\title{
Impact of tumoral carbonic anhydrase IX and Ki-67 expression on survival in oral squamous cell carcinoma patients
}

\author{
NIGEL T. BROCKTON ${ }^{1}$, PAWADEE LOHAVANICHBUTR ${ }^{2}$, EMEKA K. ENWERE ${ }^{3}$, MELISSA P. UPTON $^{4}$, \\ ELIZABETH N. KORNAGA ${ }^{3}$, STEVEN C. NAKONESHNY ${ }^{5}$, PINAKI BOSE ${ }^{5}, \mathrm{CHU} \mathrm{CHEN}^{2}$ and JOSEPH C. DORT \\ ${ }^{1}$ Department of Cancer Epidemiology and Prevention Research, Cancer Control Alberta, Alberta Health Services, Calgary, \\ AB T2S 3C3, Canada; ${ }^{2}$ Division of Public Health Sciences, Fred Hutchinson Cancer Research Center, Seattle, \\ WA 98109-1024, USA; ${ }^{3}$ Functional Tissue Imaging Unit, Translational Laboratories, Tom Baker Cancer Centre, \\ Calgary, AB T2N 4N2, Canada; ${ }^{4}$ Department of Pathology, University of Washington, Seattle, WA 98195, USA; \\ ${ }^{5}$ Ohlson Research Initiative, Arnie Charbonneau Cancer Institute, Cumming School of Medicine; ${ }^{6}$ Division of \\ Otolaryngology-Head and Neck Surgery, Department of Surgery, University of Calgary, Calgary, AB T2N 1N4, Canada
}

Received March 30, 2017; Accepted July 7, 2017

DOI: $10.3892 / 01.2017 .6829$

\begin{abstract}
Oral squamous cell carcinoma (OSCC) is the most commonly diagnosed type of head and neck cancer, accounting for $\sim 300,000$ new cases worldwide annually. Carbonic anhydrase IX (CAIX) and Ki-67 have been associated with reduced disease-specific survival (DSS) in patients with OSCC. We previously proposed a combined CAIX and Ki-67 signature of 'functional hypoxia' and sought to replicate this association in a larger independent cohort of patients with OSCC at the Fred Hutchinson Cancer Research Center (FHCRC) in Seattle. The study population included patients with incident primary OSCC treated at the University of Washington Medical Center and the Harborview Medical Center in Seattle between December 2003 and February 2012. Archived tumor blocks were obtained with tissue samples from 189 patients, and triplicate $0.6 \mathrm{~mm}$ cores were assembled into tissue microarrays (TMAs). Fluorescence immunohistochemistry and AQUAnalysis ${ }^{\circledR}$ were used to quantify the expression of tumoral CAIX (tCAIX) and stromal CAIX (sCAIX) and tumoral Ki-67 for each TMA core. Hazard ratios for DSS were calculated using Cox proportional hazards analysis. High tCAIX and sCAIX expression levels were associated with reduced DSS $(\mathrm{aHR}=1.003,95 \% \mathrm{CI}: 1.00-1.01$ and $\mathrm{aHR}=1.010$, 95\% CI:1.001-1.019, per AQUA score unit, respectively). Ki-67 expression was not associated with survival (aHR=1.01, 95\% CI:0.99-1.02) in the FHCRC cohort. DSS for patients with
\end{abstract}

Correspondence to: Dr Nigel T. Brockton, Department of Cancer Epidemiology and Prevention Research, Cancer Control Alberta, Alberta Health Services, Room 515C, 5th Floor, Holy Cross Centre, Box ACB, 2210-2nd Street. SW, Calgary, AB T2S 3C3, Canada E-mail: nigel.brockton@ahs.ca

Key words: survival, automated quantitative analysis ${ }^{\mathrm{TM}}$, carbonic anhydrase IX, Ki-67 antigen, hypoxia, proliferation, oral cancer high sCAIX and low Ki-67 did not differ from that of other patient groups. Elevated tCAIX was associated with reduced DSS as a continuous and as a dichotomized (75\%) variable. sCAIX was associated with DSS as a continuous variable but not when dichotomized (75\%). However, the previously proposed 'functional hypoxia' signature was not replicated in the current FHCRC study. The failure to replicate our prior observation of poorer survival in patients with combined high sCAIX and low tumoral Ki-67 was likely due to the absence of an association between tumoral Ki-67 and DSS in this cohort. However, the association between DSS and tCAIX and sCAIX supports a role for CAIX in OSCC clinical outcomes.

\section{Introduction}

Oral squamous cell carcinoma (OSCC) is the most commonly diagnosed cancer of the head and neck region, accounting for $\sim 300,000$ new cases annually worldwide (1). Despite advances in diagnostic, surgical and chemo-radiation techniques, the 5-year survival rates for patients with OSCC have remained at $\sim 50 \%$ for the last five decades (2). We hypothesize that biological programs, including hypoxia and proliferation, within the tumor microenvironment are associated with tumor progression and may help to identify those patients at greatest risk of relapse and mortality. Identifying those mechanisms and using those factors in clinical decision-making could help target adjuvant therapies and improve the clinical management of patients with OSCC.

Hypoxia is common within the oral tumor microenvironment but rare in normal oral epithelial tissue (3). Tumor hypoxia $\left(\mathrm{O}_{2}<5 \mathrm{mmHg}\right)$ is associated with increased invasiveness, metastasis and an overall poor prognosis (4-6). Several methods can be used to assess hypoxia within tumors. The direct measurement of tissue oxygen tension, using an oxygen electrode, is highly sensitive but intra-tumoral heterogeneity and the accessibility of tumors, due to anatomical restrictions, limit its utility for the clinical assessment of tumor hypoxia $(7,8)$. Exogenous markers, including bioreductive 
drugs (e.g., pimonidazole), enable targeted imaging but the threshold for activation by hypoxia may not correspond to clinically-relevant tumor hypoxia, and incomplete distribution due to ineffective perfusion may lead to variability in the reliability of staining across the tumor regions (6). Immunohistochemistry (IHC)-based staining for endogenous markers of hypoxia (EMH) is cost effective and routinely available in clinical pathology laboratories; however, no single EMH has been consistently demonstrated to reliably indicate an association with adverse tumor phenotypes and poor prognosis in OSCC (9).

While several hypoxic sensitizers and hypoxia-specific toxins are available as potential therapeutic agents, reliably identifying patients with hypoxic tumors for patient selection in the clinical setting is a consistent barrier to implementing effective hypoxia-targeting/sensitizing therapies (6). A number of studies have reported associations between a poorer prognosis and intratumoral levels of the EMH carbonic anhydrase IX (CAIX) in several cancer sites, including head and neck squamous cell carcinoma (10-14). CAIX expression is regulated by hypoxia inducible factor $1-\alpha$ (HIF1 $\alpha)(15)$, an oxygen-sensitive transcription factor. The stability and activity of HIF1 $\alpha$ are predominantly regulated by intracellular $\mathrm{pO}_{2}$ levels; however, elevated transcriptional activation may also occur under normoxic/mildly hypoxic conditions through the stabilization of HIF1 $\alpha$ by proliferation-inducing signaling pathways such as phosphatidylinositol 3-kinase (PI3K), nuclear factor- $\kappa \mathrm{B}(\mathrm{NF}-\kappa \mathrm{B})$, AKT and Her2 (10,16-20). Highly hypoxic tumor cells typically exhibit low proliferation $(6,21,22)$. We previously proposed that the co-localization of proliferation and EMH expression may identify cells responding to proliferative stimuli or EMH regulation by factors other than low $\mathrm{pO}_{2}$ (23), including alternative signaling pathways that control HIF1 $\alpha$ stability and activity (10,24). We had also hypothesized that functionally significant hypoxia would be characterized by low proliferation (low Ki-67 index) within areas of high stromal CAIX (sCAIX) expression (23).

Attempts to test these hypotheses led to the observations that SCAIX was more highly associated with patient survival than tumoral CAIX (tCAIX) $(13,14)$, and that there was a strong association between $\mathrm{sCAIX}{ }^{\mathrm{hi}} / \mathrm{Ki}-67^{\mathrm{lo}}$ protein levels and reduced disease-specific survival (DSS). However, the literature on prognostic biomarkers is replete with association studies that have failed independent replication (25). Therefore, the present study aimed to replicate our previous findings in an independent cohort of patients with OSCC.

\section{Materials and methods}

Patient cohort. The study cohort comprised 168 patients (mean age, 60.6 years; age range, 20-88 years; 93 males, 75 females) diagnosed with histologically confirmed incident primary OSCC treated at the University of Washington Medical Center and the Harborview Medical Center in Seattle between December 2003 and February 2012. The patients were recruited as part of a study conducted at the Fred Hutchinson Cancer Research Center (FHCRC; Seattle, WA, USA) to study genome-wide gene expression associated with the clinical outcomes of patients with OSCC of the oral cavity and oropharynx (26). The current study was conducted in accordance with the REMARK guidelines (27). The study was conducted following the acquisition of written informed consent and ethical approval from the Institutional Review Office of the Fred Hutchinson Cancer Research Center and the Conjoint Health Research Ethics Board.

Tissue microarray (TMA) construction. Formalin-fixed paraffin-embedded tumor tissue blocks suitable for analysis, as judged by a pathologist according to tumor content, were obtained for all 168 patients. TMAs were constructed using triplicate $0.6 \mathrm{~mm}$ cores from the archived tumor blocks at the FHCRC, and the sections were then shipped to the University of Calgary for IHC staining. Eleven samples of normal oral cavity squamous epithelium from control participants were incorporated in the TMAs as reference samples. Control participants were patients without OSCC who had oral surgery, including tonsillectomy or uvulopalatopharyngoplasty, at the University of Washington Medical Center and the Harborview Medical Center in Seattle between December 2003 and February 2012. Fluorescence IHC and the HistoRx ${ }^{\mathrm{TM}}$ Automated Quantitative Analysis (AQUA ${ }^{\mathrm{TM}}$ ) platform AQUAnalysis ${ }^{\circledR}$ software (Genoptix Medical Laboratory, Carlsbad, CA, USA) were used to quantify the expression of CAIX and Ki-67 for each TMA core.

Quantitative fluorescence IHC. The details of the quantitative fluorescence IHC staining conditions for CAIX, vimentin (14) and Ki-67 (28) have been described previously. Briefly, the TMA slides were stained on a DAKO AutoStainer Link 48 using antibodies against vimentin (mouse monoclonal; dilution, 1:5,000; catalog no. M0725; Dako; Agilent Technologies, Inc., Santa Clara, CA, USA), CAIX (rabbit polyclonal; dilution, 1:5,000; catalog no. ab15086; Abcam, Cambridge, MA, USA) and pan-cytokeratin (PCK; guinea pig polyclonal; dilution, 1:100; catalog no. BP5069; OriGene Technologies, Inc., Herford, Germany). For Ki-67, the MIB1 mouse monoclonal antibody was used (dilution, 1:7,000; catalog no. M724029-2; Dako; Agilent Technologies, Inc.). Slides were incubated with primary antibodies for $30 \mathrm{~min}$ at room temperature. The respective anti-mouse (catalog no. K400111-2) or anti-rabbit (catalog no. K400311-2) secondary antibody polymers (both, EnVision+ System horseradish peroxidase-labelled polymer; undiluted; Agilent Technologies Inc.) were applied for $30 \mathrm{~min}$ at room temperature followed by $5 \mathrm{~min}$ incubations, again at room temperature, with the following fluorophores: Tyramide-TSA-Plus cysteine (Cy)3 signal amplification reagent (catalog no. NEL744B001KT; PerkinElmer Inc., Waltham, MA, USA), for vimentin detection, and tyramide-Cy5 (TSA-Plus Cy5 signal amplification reagent; catalog no. NEL745B001KT; PerkinElmer Inc.), for CAIX and Ki-67 detection. For PCK detection, slides were incubated with an Alexa 488-conjugated goat anti-guinea pig secondary antibody (catalog no. A11073; dilution, 1:200; Thermo Fisher Scientific, Inc., Waltham, MA, USA) for $30 \mathrm{~min}$ at room temperature. All slides were mounted in ProLong Gold Antifade mountant containing DAPI (catalog no. P36935; Thermo Fisher Scientific, Inc.). Slides were scanned using an Aperio ScanScope ${ }^{\circledR}$ FL (Leica Microsystems, Inc., Buffalo Grove, IL, USA) and analyzed using AQUAnalysis ${ }^{\circledR}$ software (version 2.2.1.7). The tumor compartment was defined by the PCK-positive area for each 
TMA core. The tumor nuclear compartment was defined by the DAPI-positive area within the PCK-positive (tumor) compartment. The Ki-67 compartment was defined by the Ki-67-positive area within the DAPI-positive tumor nuclear compartment (28). Finally, the stromal compartment was defined by the combination of the vimentin-positive and PCK-negative areas (29). AQUAnalysis ${ }^{\circledR}$ software was used to determine the AQUA scores for sCAIX and tCAIX, as described previously (30). Ki-67 expression was calculated from the percentage of the tumor nuclear area (PCK-positive and DAPI-positive) that was Ki-67-positive (28).

Basal Ki-67 expression was defined as the lowest level measured amongst triplicate TMA cores for each patient with OSCC; this definition of Ki-67 status was identified to provide the strongest association with 5-year DSS in a prior comparison of Ki-67 scoring strategies (28). Conversely, CAIX expression for a given patient was defined using the TMA core that expressed the maximum level of sCAIX among the corresponding triplicate TMA cores. SCAIX was dichotomized at the 75th percentile to identify patients with top-quartile CAIX expression levels in the stromal compartment (sCAIX ${ }^{\mathrm{hi}}$ ) (14). Basal Ki-67 expression was dichotomized (percentage of the tumor nuclear area positive for Ki-67) at the median to define the low and high proliferation groups for the proposed replication of the combined Ki-67-sCAIX signature.

Statistical analysis. The Chi-squared test was used to compare clinical and demographic characteristics between the Calgary and FHCRC cohorts (Table I). The Kaplan-Meier (KM) estimator method and Cox's proportional hazards analyses were conducted to assess the association between expression of Ki-67, tCAIX, sCAIX and DSS of patients with OSCC. Univariate Cox proportional hazards analyses were conducted to assess the associations between $\mathrm{Ki}-67$, sCAIX, tCAIX and DSS, as categorical and as continuous variables. Survival was measured from the date of diagnosis to the date of mortality or last follow-up. Data are presented as number of patients and percentage, except for age (mean and standard deviation) (Table I). KM results are presented in survival curves (Fig. 2). Cox proportional hazards regression results are presented by hazard ratio (HR) and 95\% confidence interval (CI; Table II). The threshold for statistical significance was set at $\mathrm{P}<0.05$ or where CIs excluded unity. Multivariable Cox proportional hazards analyses were conducted to assess the impact of clinically significant covariates, including age, sex and American Joint Committee on Cancer stage. All statistical analyses (KM, Cox proportional hazards and $\chi^{2}$ ) were performed using Stata 14 (StataCorp LP, College Station, TX, USA). Optimized threshold values for Ki-67, sCAIX and tCAIX were determined using the X-Tile software ${ }^{\circledR}$ (version 3.6.1; Rimm Labs, New Haven, CT, USA) (31).

\section{Results}

Cohort characteristics. The mean age for patients included in the present study was 60.3 years [standard deviation (SD), 14.0; range, 20-88 years]. The median follow-up time for the cohort was 33 months (range, 0.2-111.0 months). The results of univariate analyses of cohort clinicopathological characteristics are presented in Table I.
Biomarker expression analysis. CAIX and Ki-67 expression levels were successfully quantified for 168/189 tumors; the data on the remaining 21 tumors were absent due to tissue processing defects, such as folded sections, and insufficient quantities of tumor tissue identified during quality control for each individual TMA core image. Images representing typical fluorescence IHC patterns for each biomarker are presented in Fig. 1. CAIX staining intensity was highest in the membranous or cytoplasmic regions in the tumor and stromal compartments (Fig. 1A). Tumor Ki-67 expression was predominantly restricted to the nucleus (Fig. 1B). Proliferative (Ki-67-positive) cells were observed in the tumoral and stromal compartments.

sCAIX and tCAIX were observed to be associated with reduced DSS (Table II). Elevated sCAIX was associated with DSS when analyzed as a continuous variable in univariate and adjusted analyses (HR, 1.014 and 95\% CIs, 1.003-1.024; HR, 1.010 and $95 \%$ CIs, 1.001-1.019, respectively). Additionally, elevated tCAIX was associated with reduced DSS when analyzed as a continuous variable in univariate and adjusted analyses (HR, 1.003 and 95\% CIs, 1.001-1.006; HR, 1.002 and 95\% CIs, 1.000-1.0005, respectively). High tCAIX was also observed to be associated with reduced DSS in univariate and adjusted analyses when dichotomized at the 75th percentile (HR, 2.19 and 95\% CIs, 1.25-3.83; HR, 1.99 and 95\% CIs, 1.13-3.50, respectively). The clinical and demographical characteristics of patients in the tCAIX ${ }^{\text {hi }}$ group did not differ significantly from those in the tCAIX ${ }^{\mathrm{lo}}$ group, for the majority of the parameters. However, those in the tCAIX ${ }^{\mathrm{hi}}$ group were more likely to have larger tumors (higher pathological tumor stage) and have nodal metastases (positive pathological nodal status; Table III). sCAIX was not significantly associated with reduced DSS when dichotomized at the 75th percentile. In contrast to our prior reports $(23,28)$, low basal Ki-67 was not associated with reduced DSS when analyzed as a continuous variable in univariate or adjusted analyses (adjusted HR, 1.01; 95\% CIs, 0.99-1.02).

Subsequently, exploratory analyses were conducted to determine an optimized threshold value for Ki-67, sCAIX and tCAIX using the X-Tile software ${ }^{\circledR}(28)$; the optimized threshold value is the threshold of Ki-67, sCAIX and tCAIX that is associated with the greatest difference in the HR for DSS between the dichotomized groups. Basal Ki-67 values ranged from 0.9-79.3 (mean, 26.5; SD, 17.7; median, 23.7), and the optimized cut-off point was set at 48.5. Applying this cut-off point suggested an increased risk (adjusted HR, 2.17, 95\% CIs, 1.08-4.38) associated with high Ki-67 (>48\% tumor nuclear area; Fig. 2A and B). Only 19/168 patients (11.3\%) exhibited Ki-67 positivity in $>48.5 \%$ of the nuclear area. tCAIX values ranged from 6.1-377.1 (mean, 98.0; SD, 92.7; median, 63.2). The optimal cut-off point for tCAIX was at 176.7 (adjusted HR, 2.45; 95\% CIs, 1.40-4.31); 33/168 tumors $(\sim 20 \%)$ expressed tCAIX above this value (Fig. $2 \mathrm{C}$ and D). sCAIX values ranged from 4.8-127.58 (mean, 28.6; SD, 23.3; median, 20.8). The optimal cut-off point for sCAIX was set at 54.8 (adjusted HR, 2.39 95\% CIs, 1.22-4.67; Fig. 2E and F); $17 / 168$ tumors $(\sim 10 \%)$ expressed sCAIX above this value.

Non-replication of the 'functional hypoxia' (combined sCAIX and Ki-67 expression) signature. In our prior report (23), 
Table I. Comparison between clinicopathological and demographical characteristics of the Calgary and FHCRC cohorts.

\begin{tabular}{|c|c|c|c|c|c|}
\hline \multirow[b]{2}{*}{ Patient characteristics } & \multicolumn{2}{|c|}{ Calgary (n=121) } & \multicolumn{2}{|c|}{ FHCRC $(n=168)$} & \multirow{2}{*}{$\begin{array}{c}{ }^{\mathrm{a}} \chi^{2} \\
\text { P-value }\end{array}$} \\
\hline & $\mathrm{n}$ & $\%$ & $\mathrm{n}$ & $\%$ & \\
\hline Sex & & & & & 0.03 \\
\hline Male & 82 & 67.8 & 93 & 55.4 & \\
\hline Female & 39 & 32.2 & 75 & 44.6 & \\
\hline Age & & & & & \\
\hline Years (mean, SD) & $60.6(13.5)$ & & $60.3(14.0)$ & & \\
\hline History of tobacco smoking & & & & & 0.34 \\
\hline Never & 28 & 23.1 & 48 & 28.6 & \\
\hline Ever & 91 & 75.2 & 120 & 71.4 & \\
\hline Missing & 2 & 1.7 & & & \\
\hline History of alcohol consumption & & & & & 0.39 \\
\hline Never & 13 & 10.7 & 21 & 12.5 & \\
\hline Ever & 65 & 53.7 & 146 & 86.9 & \\
\hline Missing & 43 & 35.5 & 1 & 0.6 & \\
\hline pT status & & & & & 0.80 \\
\hline pT1/pT2 (low) & 70 & 57.9 & 96 & 57.1 & \\
\hline pT3/pT4 (high) & 48 & 39.7 & 70 & 41.7 & \\
\hline Missing & 3 & 2.5 & 2 & 1.2 & \\
\hline pN status & & & & & 0.10 \\
\hline N0 neck & 75 & 62.0 & 88 & 52.4 & \\
\hline $\mathrm{N}+$ neck & 46 & 38.0 & 80 & 47.6 & \\
\hline Tumor differentiation & & & & & \\
\hline Well & 18 & 14.9 & 35 & 20.8 & 0.36 \\
\hline Moderate & 60 & 49.6 & 90 & 53.6 & \\
\hline Poor & 14 & 11.6 & 34 & 20.2 & \\
\hline Missing & 29 & 24.0 & 9 & 5.4 & \\
\hline Oral cavity subsite & & & & & 0.10 \\
\hline Oral tongue & 49 & 40.5 & 63 & 37.5 & \\
\hline Floor of mouth & 32 & 26.4 & 39 & 23.2 & \\
\hline Buccal & 17 & 14.0 & 14 & 8.3 & \\
\hline Gingiva & 13 & 10.7 & 37 & 22.0 & \\
\hline Others/site NOS & 10 & 8.3 & 15 & 8.9 & \\
\hline Treatment & & & & & a \\
\hline Surgery alone & 38 & 31.4 & 69 & 41.1 & \\
\hline Surgery + RT & 83 & 68.6 & 40 & 23.8 & \\
\hline Surgery + chemo + RT & & & 52 & 31.0 & \\
\hline Surgery + chemo & & & 2 & 1.2 & \\
\hline Incomplete data & & & 5 & 3.0 & \\
\hline
\end{tabular}

${ }^{a}$ Missing data were excluded from the $\chi^{2}$ test. ${ }^{b}$ Not calculated. FHCRC, Fred Hutchinson Cancer Research Centre; RT, radiotherapy; NOS, not otherwise specified.

stratification of $\mathrm{sCAIX}{ }^{\mathrm{hi}}$-expressing tumors according to their median basal Ki-67 expression identified patients with significantly poorer 5-year DSS (HR, 7.2; 95\% CIs, 2.5-20.4). However, tumors defined by combined sCAIX ${ }^{\text {hi }}$ and $\mathrm{Ki}-67^{\mathrm{lo}}$ were not associated with reduced 5-year DSS in the FHCRC cohort in univariate or adjusted analyses (HR, 1.43 and 95\% CIs, 0.69-2.97; HR, 1.18 and 95\% CIs, 0.59-2.41, respectively).

\section{Discussion}

Our previous study demonstrated, in two independent cohorts, that SCAIX was more highly associated with patient survival than tCAIX $(13,14)$. In the current study, elevated sCAIX and tCAIX were associated with reduced DSS when analyzed as continuous variables, but only tCAIX was associated with DSS when dichotomized at the 75 th percentile. The association 
Table II. Summary of univariate and multivariate Cox proportional hazards analysis of OSCC DSS.

\begin{tabular}{llc}
\hline Biomarker & \multicolumn{1}{c}{ Analysis } & Hazard ratios, (95\% CIs) \\
\hline sCAIX (continuous) & Univariate & $1.014,(1.003-1.024)$ \\
& Adjusted (age, sex and stage) & $1.010,(1.001-1.019)$ \\
tCAIX (continuous) & Univariate & $1.003,(1.001-1.006)$ \\
& Adjusted (age, sex and stage) & $1.002,(1.000-1.0005)$ \\
Ki67 (continuous) & Univariate & $1.009,(0.995-1.023)$ \\
& Adjusted (age, sex and stage) & $1.009,(0.995-1.024)$ \\
tCAIX (dichotomized, 75\%) & Univariate & $2.19,(1.25-3.83)$ \\
& Adjusted (age, sex and stage) & $1.99,(1.13-3.50)$ \\
sCAIX (dichotomized, 75\%) & Univariate & $1.36,(0.74-2.51)$ \\
& Adjusted (age, sex and stage) & $1.23,(0.68-2.23)$ \\
Ki67 (dichotomized, median) & Univariate & $1.23,(0.73-2.06)$ \\
& Adjus.ted (age, sex and stage) & $1.29,(0.75-2.21)$
\end{tabular}

OSCC, oral squamous cell carcinoma; CAIX, carbonic anhydrase IX; s, stromal; t, tumoral; CI, confidence interval; DSS, disease-specific survival.

A

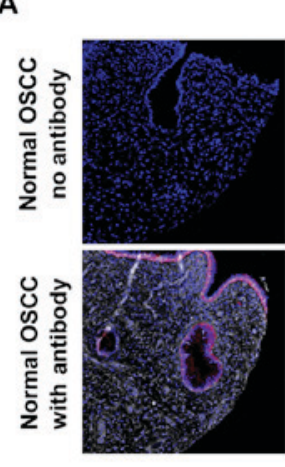

C

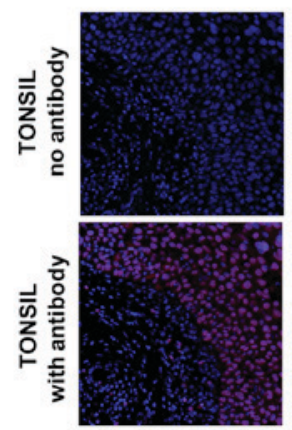

B

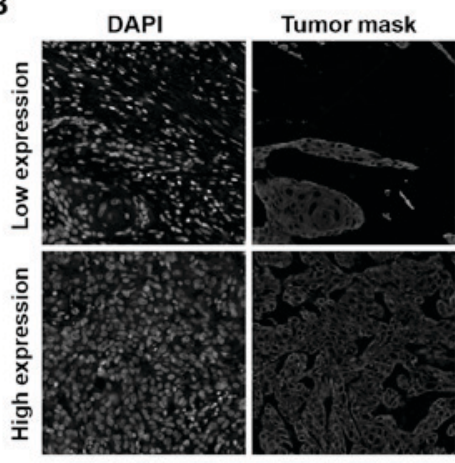

Green

D

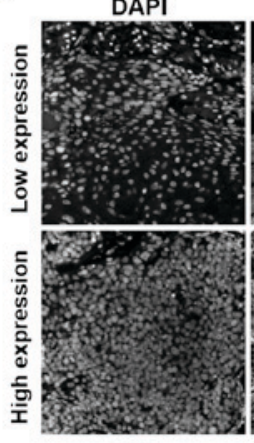

Blue

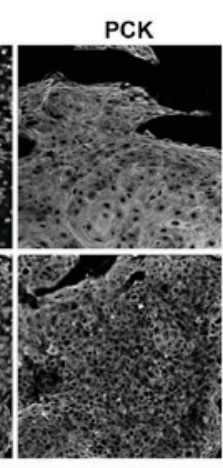

Green

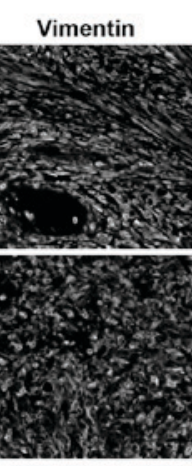

Grey

Ki-67

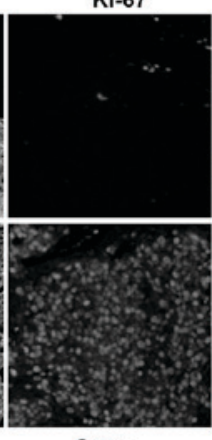

Grey

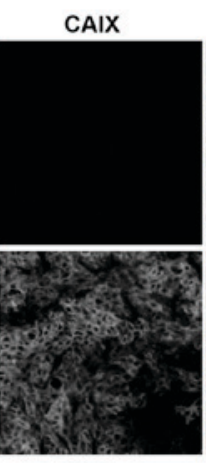

Red

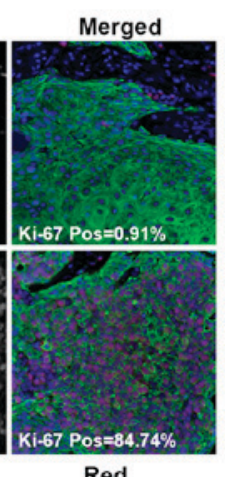

Figure 1. Fluorescent immunohistochemical staining imaged with an Aperio ScanScope FL ${ }^{\circledast}$ and analyzed using AQUAnalysis ${ }^{\circledR}$ software. Indicated are representative fluorescence immunohistochemistry images of (A and B) CAIX and (C and D) Ki-67 staining in OSCC samples. (A) Positive and negative controls for CAIX involved the staining of normal OSCE with/and without the CAIX antibody, respectively. (C) Similarly, positive and negative controls for Ki-67 involved the staining of tonsil tissue with or without the Ki-67 antibody, respectively. Representative images of OSCC samples stained for (B) CAIX and (D) Ki-67 are indicated with examples of high and low expressers of these proteins. Merged images are pseudo-colored blue for DAPI, green for pan-cytokeratin, red for the biomarker of interest and grayscale for vimentin. OSCE, oral squamous cell epithelium; OSCC, oral squamous cell carcinoma; AQUA, Automated Quantitative Analysis ${ }^{\mathrm{TM}}$; CAIX, carbonic anhydrase IX; s, stromal.

between SCAIX and DSS, when analyzed as a continuous variable but not as a categorical variable, suggests that sCAIX may be a biologically important factor in OSCC survival but also that the $75 \%$ cut-off point is not appropriate for clinical decision making.
The association between DSS and SCAIX and tCAIX, for each unit increase in expression (AQUA score), was similar (sCAIX: HR, 1.014, 95\% CIs, 1.003-1.024 vs. tCAIX: HR, $1.003,95 \%$ CIs, 1.001-1.006). The increased risks associated with each measure of CAIX expression were also similar when 
Table III. Comparison of clinical and demographic features between patient groups stratified by tCAIX ( $<75$ th vs. $\geq 75$ th percentiles).

\begin{tabular}{|c|c|c|c|c|c|}
\hline \multirow[b]{2}{*}{ Patient characteristics } & \multicolumn{2}{|c|}{ Low tCAIX (n=126) } & \multicolumn{2}{|c|}{ High tCAIX $(n=42)$} & \multirow{2}{*}{$\begin{array}{c}\chi^{2} \\
\text { P-value }\end{array}$} \\
\hline & $\mathrm{n}$ & $\%$ & $\mathrm{n}$ & $\%$ & \\
\hline Sex & & & & & 0.929 \\
\hline Male & 70 & 55.6 & 23 & 54.8 & \\
\hline Female & 56 & 44.4 & 19 & 45.2 & \\
\hline \multicolumn{6}{|l|}{ Age } \\
\hline Years (mean, SD) & $60.8(14.4)$ & & $58.5(13.0)$ & & \\
\hline History of tobacco smoking & & & & & 0.115 \\
\hline Never & 32 & 25.4 & 16 & 38.1 & \\
\hline Ever & 94 & 74.6 & 26 & 61.9 & \\
\hline History of alcohol consumption & & & & & 0.144 \\
\hline Never & 13 & 10.3 & 8 & 19.1 & \\
\hline Ever & 112 & 88.9 & 34 & 80.9 & \\
\hline Missing & 1 & 0.8 & & & \\
\hline pT status & & & & & $0.019^{\mathrm{a}}$ \\
\hline pT1/pT2 (low) & 80 & 63.5 & 18 & 42.9 & \\
\hline pT3/pT4 (high) & 46 & 36.5 & 24 & 57.1 & \\
\hline Missing & 0 & 0.0 & 0 & 0.0 & \\
\hline pN status & & & & & $0.004^{\mathrm{a}}$ \\
\hline N0 neck & 74 & 58.7 & 14 & 33.3 & \\
\hline $\mathrm{N}+$ neck & 52 & 41.3 & 28 & 66.7 & \\
\hline Tumor differentiation & & & & & 0.170 \\
\hline Well & 30 & 23.8 & 5 & 11.9 & \\
\hline Moderate & 64 & 50.8 & 26 & 61.9 & \\
\hline Poor & 23 & 18.3 & 11 & 26.2 & \\
\hline Missing & 9 & 7.1 & 0 & 0.0 & \\
\hline Oral cavity subsite & & & & & 0.068 \\
\hline Oral tongue & 46 & 36.5 & 17 & 40.5 & \\
\hline Floor of mouth & 25 & 19.8 & 14 & 33.3 & \\
\hline Buccal & 9 & 7.1 & 5 & 11.9 & \\
\hline Gingiva & 32 & 25.4 & 5 & 11.9 & \\
\hline Others/site NOS & 14 & 11.1 & 1 & 2.4 & \\
\hline Treatment & & & & & $0.032^{\mathrm{a}}$ \\
\hline Surgery alone & 58 & 46.0 & 11 & 26.2 & \\
\hline Surgery + RT & 31 & 24.6 & 9 & 21.4 & \\
\hline Surgery + chemo + RT & 32 & 25.4 & 20 & 47.6 & \\
\hline Surgery + chemo & 1 & 0.8 & 1 & 2.4 & \\
\hline Incomplete data & 4 & 3.2 & 1 & 2.4 & \\
\hline
\end{tabular}

${ }^{\mathrm{a}} \mathrm{P}<0.05$. OSCC, oral squamous cell carcinoma; CAIX, carbonic anhydrase IX; s, stromal; t, tumoral; CI, confidence interval; DSS, disease-specific survival; RT, radiotherapy; NOS, not otherwise specified.

dichotomized at their optimized cut points (sCAIX adjusted HR, 2.39, 95\% CIs, 1.22-4.67; tCAIX adjusted HR, 2.45, 95\% CIs, 1.40-4.31). However, the group defined by the optimized cut-off point for tCAIX contained almost twice as many patients, compared with the group defined by the optimized cut-off point for sCAIX. The strength of the association with DSS was similar for each dichotomized measure of CAIX, suggesting that, tCAIX is a more informative prognostic marker than sCAIX. Determining which of the two, tCAIX or sCAIX, is the more informative measure of tumor CAIX status and defining a biologically meaningful expression threshold remains a necessary step if this marker is to be translated into a clinically useful test.

Our prior study reported the prognostic impact of a combined sCAIX/Ki-67 signature on DSS in OSCC (23). This signature was proposed on the basis of the potential 
A

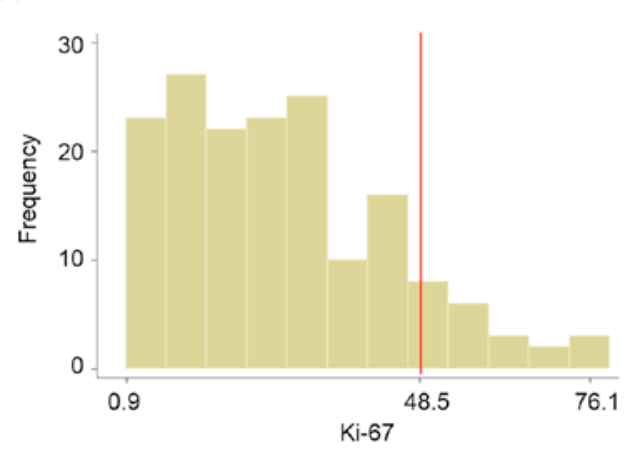

B

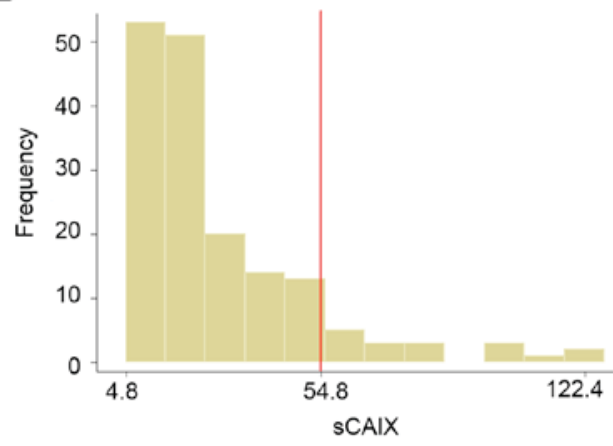

C

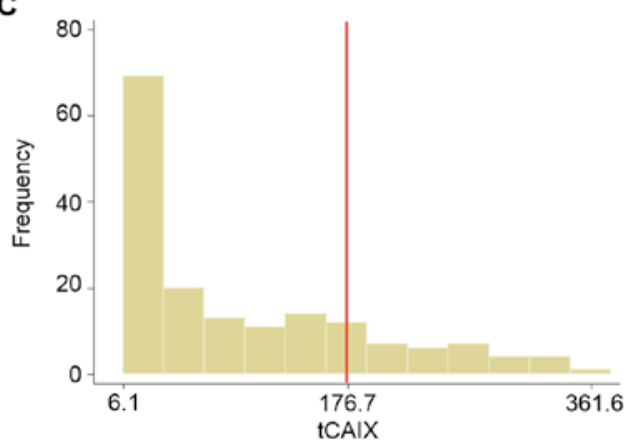

D

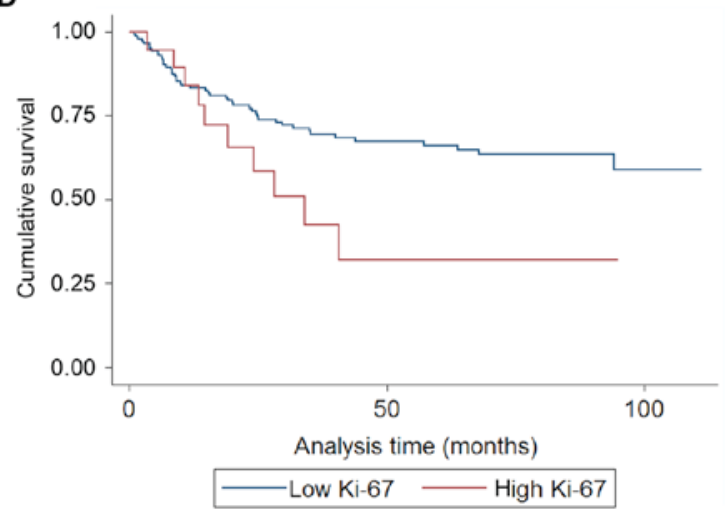

E

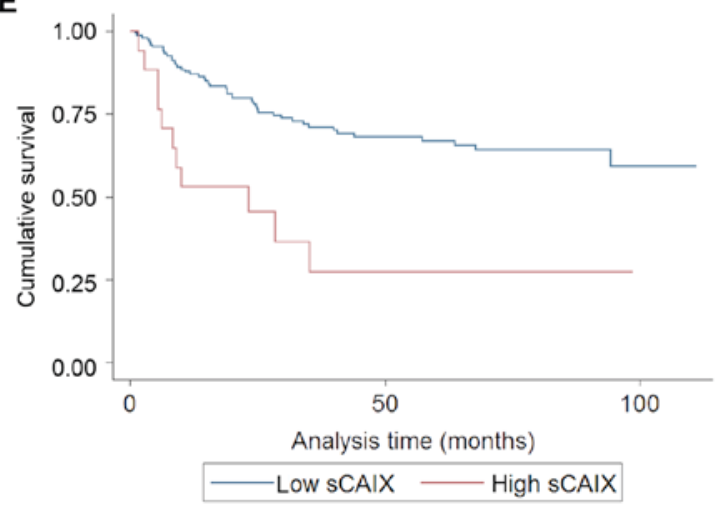

F

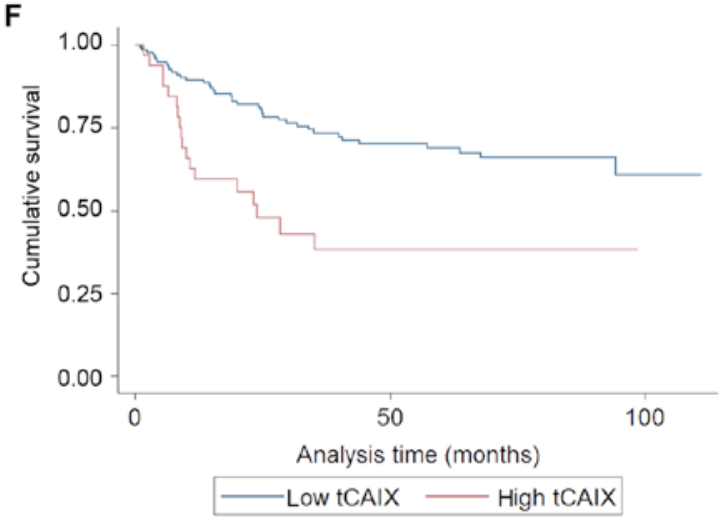

Figure 2. Frequency distributions of AQUA ${ }^{\circledast}$ scores in OSCC patients and Kaplan-Meier survival curves according to the optimized AQUA ${ }^{\circledast}$ score cut-off points derived using X-Tile ${ }^{\circledR}$ software. (A) Frequency distribution histogram for Ki-67 AQUA ${ }^{\circledR}$ scores. (B) Frequency distribution histogram for sCAIX AQUA $^{\circledast}$ scores. (C) Frequency distribution histogram for tCAIX AQUA ${ }^{\circledast}$ scores. (D) Kaplan-Meier survival curve according to optimized threshold value for Ki-67 AQUA ${ }^{\circledR}$ scores. (E) Kaplan-Meier survival curve according to optimized threshold value for sCAIX AQUA ${ }^{\circledR}$ scores. (F) Kaplan-Meier survival curve according to optimized threshold value for tCAIX AQUA ${ }^{\circledR}$ scores. OSCC, oral squamous cell carcinoma; AQUA, Automated Quantitative Analysis ${ }^{\mathrm{TM}}$; CAIX, carbonic anhydrase IX; t, tumoral, s, stromal.

confounding effect of proliferation, and associated signaling pathways, on the association between CAIX expression and prognosis. This was a logical extension of our previous research, reporting significantly reduced survival in patients with high sCAIX and low Ki-67, separately $(14,28)$. The current study did not replicate this proposed 'functional hypoxia' signature according to our original definitions of low Ki-67 and high sCAIX. The association between DSS and each component of the functional hypoxia signature was then further examined. The failure of each individual component may explain the lack of association detected in the proposed 'functional hypoxia' signature. In contrast to prior reports $(14,23)$, sCAIX was not significantly associated with survival when dichotomized at the 75th percentile in univariate and adjusted analyses (HR, 1.36 and 95\% CIs, 0.74-2.51; HR, 1.23 and 95\% CIs, 0.68-2.23, respectively). Furthermore, when Ki-67 was analyzed as a continuous variable, there was no association with reduced survival in univariate or adjusted analyses (HR, 1.009 and 95\% CIs, 0.995-1.023; HR, 1.009 and 95\% CIs, 0.995-1.024, respectively) in the current study.

Hypoxic tumors are typically associated with a poor prognosis; not all tumors are considered to be hypoxic, but 
a high proportion of tumors have areas of hypoxia and tumors which exhibit increased hypoxia typically have poorer outcomes $(22,32)$. The potential role for hypoxia and proliferation in the prognosis of OSCC, and other types of head and neck cancer, has been investigated by a number of research groups (33-35). Recently, there has been particular interest in the role of CAIX in the stroma as it should not be confounded by somatic gene alterations commonly observed in OSCC. For example, the von Hippel-Lindau (VHL) gene, a negative regulator of HIF1 $\alpha$ activity, is commonly deleted in OSCC (36). The loss of VHL leads to an accumulation of HIF $1 \alpha$ and drives the hypoxia-independent expression of CAIX $(37,38)$. However, proliferation-associated factors may also enhance sCAIX expression despite the absence of hypoxia. The response of tumor cells to proliferative signaling in the tumor microenvironment is likely to be most relevant to the disease course. Consequently, we assigned proliferation status of the tumor/patient based on the measurement of Ki-67 in the PCK-positive tumor cell compartment. However, paracrine/autocrine growth factor signaling within the tumor microenvironment may contribute to HIF1 $\alpha$ stabilization in the tumor stroma, and elevate SCAIX expression independent of hypoxia. Furthermore, local depletion of oxygen and nutrients due to the high tumor cell density may induce peri-cellular hypoxia and consequent elevation in sCAIX expression $(10,39)$.

The cohorts of oral cancer patients from Calgary and FHCRC are broadly similar when comparing common clinical and demographic characteristics (Table II). Therefore, the failure to replicate the previous combined Ki-67-sCAIX association cannot be solely attributed to differences in these populations. However, the limitation of the sub-group sizes and post hoc definition of survival groups in the original report is acknowledged, and emphasizes the requirement for replication in an independent OSCC cohort.

Several mRNA-based multi-parameter prognostic and predictive assays have been proposed for head and neck cancer and for other cancer types, particularly breast cancer (5,40-42). However, due to logistical and cost challenges, few gene expression-based tests are currently in routine clinical use for treatment selection. IHC-based assays, for the purposes of diagnosis and prognostication, are appealing as standard clinical pathology laboratories are equipped to conduct these assays. Recent evidence suggests that the IHC-based tests provide similar assessments of recurrence risk in patients with breast cancer, so the potential to develop an equivalent test in patients with OSCC is present (43).

The current study adhered to the REMARK criteria for the reporting of prognostic studies (27). The present study was limited by its retrospective design and the lack of corroborative measures of intra-tumoral hypoxia, such as directly measured $\mathrm{pO}_{2}$ or pimonidazole distribution. Our results support the role of CAIX in OSCC survival but the relative importance of sCAIX or tCAIX is unclear. The addition of proliferation markers, such as Ki-67, could potentially improve the prognostic utility of sCAIX or tCAIX, but the present study was unable to replicate the prior findings (23). Studies in larger cohorts of patients with OSCC could enable the derivation of prognostic signatures for OSCC or the identification of specific hypoxia-sensitizing/targeting treatments (44).

\section{Acknowledgements}

The current study was supported by a grant from the US National Cancer Institute, National Institutes of Health (grant no. RO1CA 095419), with additional funds from the Fred Hutchinson Cancer Research Center and the Ohlson Research Initiative in Head and Neck Cancer Research.

\section{References}

1. Ferlay J, Soerjomataram I, Ervik M, Dikshit R, Eser S, Mathers C, Rebelo M, Parkin DM, Forman D and Bray F: GLOBOCAN 2012 v1.0, Cancer Incidence and Mortality Worldwide: IARC CancerBase No. 11: 2016, 2016.

2. Campana JP and Meyers AD: The surgical management of oral cancer. Otolaryngol Clin North Am 39: 331-348, 2006.

3. Chen L, Gajendrareddy PK and DiPietro LA: Differential expression of HIF-1 $\alpha$ in skin and mucosal wounds. J Dent Res 91: 871-876, 2012.

4. Janssen HL, Haustermans KM, Balm AJ and Begg AC: Hypoxia in head and neck cancer: How much, how important? Head Neck 27: 622-638, 2005.

5. Toustrup K, Sørensen BS, Alsner J and Overgaard J: Hypoxia gene expression signatures as prognostic and predictive markers in head and neck radiotherapy. Semin Radiat Oncol 22: 119-127, 2012.

6. Wilson WR and Hay MP: Targeting hypoxia in cancer therapy. Nat Rev Cancer 11: 393-410, 2011.

7. Olive PL, Banáth JP and quino-Parsons C: Measuring hypoxia in solid tumours-is there a gold standard? Acta Oncol 40: 917-923, 2001.

8. Le QT: Identifying and targeting hypoxia in head and neck cancer: A brief overview of current approaches. Int J Radiat Oncol Biol Phys 69 (2 Suppl): S56-S58, 2007.

9. Oliveira LR and Ribeiro-Silva A: Prognostic significance of immunohistochemical biomarkers in oral squamous cell carcinoma. Int J Oral Maxillofac Surg 40: 298-307, 2011.

10. Kaluz S, Kaluzova M, Liao SY, Lerman M and Stanbridge EJ: Transcriptional control of the tumor- and hypoxia-marker carbonic anhydrase 9: A one transcription factor (HIF-1) show? Biochim Biophys Acta 1795: 162-172, 2009.

11. Hoogsteen IJ, Marres HA, Bussink J, van der Kogel AJ and Kaanders JH: Tumor microenvironment in head and neck squamous cell carcinomas: Predictive value and clinical relevance of hypoxic markers. Head Neck 29: 591-604, 2007.

12. McDonald PC, Winum JY, Supuran CT and Dedhar S: Recent developments in targeting carbonic anhydrase IX for cancer therapeutics. Oncotarget 3: 84-97, 2012.

13. Brockton N, Dort J, Lau H, Hao D, Brar S, Klimowicz A, Petrillo S, Diaz R, Doll C and Magliocco A: High stromal carbonic anhydrase IX expression is associated with decreased survival in P16-negative head-and-neck tumors. Int J Radiat Oncol Biol Phys 80: 249-257, 2011.

14. Brockton NT, Klimowicz AC, Bose P, Petrillo SK, Konno M, Rudmik L, Dean M, Nakoneshny SC, Matthews TW, Chandarana S, et al: High stromal carbonic anhydrase IX expression is associated with nodal metastasis and decreased survival in patients with surgically-treated oral cavity squamous cell carcinoma. Oral Oncol 48: 615-622, 2012.

15. Sowter HM, Raval RR, Moore JW, Ratcliffe PJ and Harris AL: Predominant role of hypoxia-inducible transcription factor (Hif)-1alpha versus Hif-2alpha in regulation of the transcriptional response to hypoxia. Cancer Res 63: 6130-6134, 2003.

16. BelAiba RS, Bonello S, Zähringer C, Schmidt S, Hess J, Kietzmann T and Görlach A: Hypoxia up-regulates hypoxia-inducible factor-1alpha transcription by involving phosphatidylinositol 3-kinase and nuclear factor kappaB in pulmonary artery smooth muscle cells. Mol Biol Cell 18: 4691-4697, 2007.

17. Pore N, Jiang ZB, Shu HK, Bernhard E, Kao GD and Maity A: Akt1 activation can augment hypoxia-inducible factor-1alpha expression by increasing protein translation through a mammalian target of rapamycin-independent pathway. Mol Cancer Res 4: 471-479, 2006.

18. Laughner E, Taghavi P, Chiles K, Mahon PC and Semenza GL: HER2 (neu) signaling increases the rate of hypoxia-inducible factor 1alpha (HIF-1alpha) synthesis: Novel mechanism for HIF-1-mediated vascular endothelial growth factor expression. Mol Cell Biol 21: 3995-4004, 2001. 
19. Harris AL: Hypoxia-a key regulatory factor in tumour growth. Nat Rev Cancer 2: 38-47, 2002.

20. Kaluz S, Kaluzova M, Chrastina A, Olive PL, Pastoreková S, Pastorek J, Lerman MI and Stanbridge EJ: Lowered oxygen tension induces expression of the hypoxia marker MN/carbonic anhydrase IX in the absence of hypoxia-inducible factor 1 alpha stabilization: A role for phosphatidylinositol 3'-kinase. Cancer Res 62: 4469-4477, 2002.

21. Sullivan R and Graham CH: Hypoxia-driven selection of the metastatic phenotype. Cancer Metastasis Rev 26: 319-331, 2007.

22. Jubb AM, Buffa FM and Harris AL: Assessment of tumour hypoxia for prediction of response to therapy and cancer prognosis. J Cell Mol Med 14: 18-29, 2010.

23. Klimowicz AC, Bose P, Petrillo SK, Magliocco AM, Dort JC and Brockton NT: The prognostic impact of a combined carbonic anhydrase IX and Ki67 signature in oral squamous cell carcinoma. Br J Cancer 109: 1859-1866, 2013.

24. Pastorekova S, Ratcliffe PJ and Pastorek J: Molecular mechanisms of carbonic anhydrase IX-mediated $\mathrm{pH}$ regulation under hypoxia. BJU Int 101 (Suppl 4): S8-S15, 2008.

25. Søland TM and Brusevold IJ: Prognostic molecular markers in cancer-quo vadis? Histopathology 63: 297-308, 2013.

26. Chen C, Méndez E, Houck J, Fan W, Lohavanichbutr P, Doody D, Yueh B, Futran ND, Upton M, Farwell DG, et al: Gene expression profiling identifies genes predictive of oral squamous cell carcinoma. Cancer Epidemiol Biomarkers Prev 17: 2152-2162, 2008.

27. McShane LM, Altman DG, Sauerbrei W, Taube SE, Gion M and Clark GM; Statistics Subcommittee of the NCI-EORTC Working Group on Cancer Diagnostics: Reporting recommendations for tumor marker prognostic studies. J Clin Oncol 23: 9067-9072, 2005

28. Klimowicz AC, Bose P, Nakoneshny SC, Dean M, Huang L, Chandarana S, Magliocco AM, Wayne Matthews T, Brockton NT and Dort JC: Basal Ki67 expression measured by digital image analysis is optimal for prognostication in oral squamous cell carcinoma. Eur J Cancer 48: 2166-2174, 2012.

29. Bose P, Klimowicz AC, Kornaga E, Petrillo SK, Matthews TW, Chandarana S, Magliocco AM, Brockton NT and Dort JC: Bax expression measured by AQUAnalysis is an independent prognostic marker in oral squamous cell carcinoma. BMC Cancer 12: $332,2012$.

30. Gustavson MD, Bourke-Martin B, Reilly DM, Cregger M, Williams C, Tedeschi G, Pinard R and Christiansen J: Development of an unsupervised pixel-based clustering algorithm for compartmentalization of immunohistochemical expression using automated QUantitative Analysis. Appl Immunohistochem Mol Morphol 17: 329-337, 2009.

31. Camp RL, Dolled-Filhart M and Rimm DL: X-tile: A new bio-informatics tool for biomarker assessment and outcome-based cut-point optimization. Clin Cancer Res 10: 7252-7259, 2004.

32. Vaupel P and Mayer A: Hypoxia in cancer: Significance and impact on clinical outcome. Cancer Metastasis Rev 26: 225-239, 2007.

33. Ljungkvist AS, Bussink J, Rijken PF, Kaanders JH, van der Kogel AJ and Denekamp J: Vascular architecture, hypoxia, and proliferation in first generation xenografts of human head-and-neck squamous cell carcinomas. Int J Radiat Oncol Biol Phys 54: 215-228, 2002.
34. Hoogsteen IJ, Marres HA, Wijffels KI, Rijken PF, Peters JP, van den Hoogen FJ, Oosterwijk E, van der Kogel AJ and Kaanders JH: Colocalization of carbonic anhydrase 9 expression and cell proliferation in human head and neck squamous cell carcinoma. Clin Cancer Res 11: 97-106, 2005.

35. Kondo Y, Yoshikawa K, Omura Y, Shinohara A, Kazaoka Y, Sano J, Mizuno Y, Yokoi T and Yamada S: Clinicopathological significance of carbonic anhydrase 9, glucose transporter-1, Ki-67 and p53 expression in oral squamous cell carcinoma. Oncol Rep 25: 1227-1233, 2011.

36. Sparano A, Quesnelle KM, Kumar MS, Wang Y, Sylvester AJ, Feldman M, Sewell DA, Weinstein GS and Brose MS: Genome-wide profiling of oral squamous cell carcinoma by array-based comparative genomic hybridization. Laryngoscope 116: 735-741, 2006.

37. Wykoff CC, Beasley NJ, Watson PH, Turner KJ, Pastorek J, Sibtain A, Wilson GD, Turley H, Talks KL, Maxwell PH, et al: Hypoxia-inducible expression of tumor-associated carbonic anhydrases. Cancer Res 60: 7075-7083, 2000.

38. Ivanov SV, Kuzmin I, Wei MH, Pack S, Geil L, Johnson BE, Stanbridge EJ and Lerman MI: Down-regulation of transmembrane carbonic anhydrases in renal cell carcinoma cell lines by wild-type von Hippel-Lindau transgenes. Proc Natl Acad Sci USA 95: 12596-12601, 1998.

39. Chrastina A: High cell density-mediated pericellular hypoxia is a crucial factor inducing expression of the intrinsic hypoxia marker CA IX in vitro in HeLa cells. Neoplasma 50: 251-256, 2003.

40. Ross JS, Hatzis C, Symmans WF, Pusztai L and Hortobágyi GN: Commercialized multigene predictors of clinical outcome for breast cancer. Oncologist 13: 477-493, 2008.

41. Nielsen TO, Parker JS, Leung S, Voduc D, Ebbert M, Vickery T, Davies SR, Snider J, Stijleman IJ, Reed J, et al: A comparison of PAM50 intrinsic subtyping with immunohistochemistry and clinical prognostic factors in tamoxifen-treated estrogen receptor-positive breast cancer. Clin Cancer Res 16: 5222-5232, 2010.

42. Cronin M, Sangli C, Liu ML, Pho M, Dutta D, Nguyen A, Jeong J, $\mathrm{Wu}$ J, Langone $\mathrm{KC}$ and Watson D: Analytical validation of the oncotype DX genomic diagnostic test for recurrence prognosis and therapeutic response prediction in node-negative, estrogen receptor-positive breast cancer. Clin Chem 53: 1084-1091, 2007.

43. Bartlett JM, Bayani J, Marshall A, Dunn JA, Campbell A, Cunningham C, Sobol MS, Hall PS, Poole CJ, Cameron DA, et al: Comparing breast cancer multiparameter tests in the OPTIMA prelim trial: No test is more equal than the others. J Natl Cancer Inst 108: pii: djw050, 2016.

44. Chi JT, Wang Z, Nuyten DS, Rodriguez EH, Schaner ME, Salim A, Wang Y, Kristensen GB, Helland A, Børresen-Dale AL, et al: Gene expression programs in response to hypoxia: Cell type specificity and prognostic significance in human cancers. PLoS Med 3: e47, 2006. 